\title{
Hemangiossarcoma cutâneo paravertebral em cão causando compressão medular
}

\section{Paravertebral cutaneous hemangiosarcoma in dog causing medular compression}

\author{
Rogério Anderson Marcasso ${ }^{1^{*}}$; Mônica Vicky Bahr Arias ${ }^{2 *}$; \\ Paula Cava Rodrigues ${ }^{3}$; Ana Paula Frederico Rodrigues Loureiro Bracarense ${ }^{4}$
}

\begin{abstract}
Resumo
Um cão macho, scottish terrier, de sete anos foi atendido no Hospital Veterinário da Universidade Estadual de Londrina por apresentar paraplegia grau $\mathrm{V}$ e um nódulo em região dorso lombar direita de crescimento lento, com evolução de dois meses. Foi realizado mielografia, visibilizando-se interrupção na coluna de contraste entre as vértebras torácicas $11^{\mathrm{a}}$ e $12^{\mathrm{a}}$. Assim, procedeu-se à hemilaminectomia nesta região, não sendo constatado compressão medular, procedendo-se a ampliação caudal da abertura da lâmina vertebral T12. Na região da quarta vértebra lombar observou-se um desvio da medula espinhal para o lado esquerdo devido à presença de uma massa de coloração avermelhada proveniente do lado direito, diagnosticando-se infiltração tumoral em vértebras com compressão medular, não sendo possível sua remoção cirúrgica. Na histologia classificou-se o tumor como hemangiossarcoma. Este relato enfatiza a importância de considerar a possibilidade de neoplasias no diagnóstico diferencial de paraplegias, mesmo em alterações clínicas agudas.
\end{abstract}

Palavras-chave: Hemangiossarcoma, paraplegia, síndrome toracolombar, cão

\begin{abstract}
A seven-year-old male scottish terrier was examined at the Veterinary Hospital of the Universidade Estadual de Londrina due to a toracolumbar syndrome classified as V degree and a mass in lumbar region back right of slow growth with evolution of two months. Myelography showed an interruption of the column of contrast between the $11^{\text {th }}$ and $12^{\text {th }}$ thoracic vertebrae. A hemilaminectomy was performed in this region. Spinal cord compression at this location was not observed, however during the caudal enlargement of hemilaminectomy it was visualized in the region of the fourth lumbar vertebrae, a spinal cord deviation to the left, due to the presence of a reddish mass at the right side that was diagnosed as a tumor infiltration in the vertebrae with cord compression. Surgical removal with appropriate margin was not possible. In histology, the tumor was classified as hemangiosarcoma. This report emphasizes the importance of considering the possibility of cancer as differential diagnosis of paraplegias, even in acute clinical changes.
\end{abstract}

Key words: Hemangiosarcoma, paraplegia, toracolumbar syndrome, dog

\footnotetext{
Mestrando em Ciência Animal, Universidade Estadual de Londrina. E-mail: romarcasso@yahoo.com.br

2 Professora Associada, Departamento de Clínicas Veterinárias, Universidade Estadual de Londrina. E-mail: vicky@uel.br

3 Médica Veterinária autônoma. E-mail: paulacava2@hotmail.com

4 Professora Associada, Departamento de Medicina Veterinária Preventiva, Universidade Estadual de Londrina, E-mail: anapaula@, uel.br

* Autor para correspondência
} 


\section{Introdução}

O hemangiossarcoma é uma neoplasia maligna de origem endotelial vascular que acomete preferencialmente o baço, fígado e átrio direito, (BROWN; PATNAIL; MACEWEN, 1985; LORIMIER; KITCHELL, 2002; SMITH, 2003) sendo a forma cutânea mais rara (SIMON, 2006; MACEWEN, 2007). O hemangiossarcoma cutâneo equivale a $14 \%$ de todos os casos de hemangiossarcoma e a menos de $0,2 \%$ de todas as neoplasias malignas (CHUN, 1999). A etiologia é desconhecida (MACEWEN, 2007), porém relata-se a associação com a exposição solar e predisposição genética (LORIMIER; KITCHELL, 2002). As raças mais acometidas são pastores alemães e poodles (BROWN, PATNAIL; MACEWEN, 1985), porém, o hemangiossarcoma cutâneo tende a ocorrer principalmente em cães de pelame curto, como whippets, pit bulls, boxers, greyhound e dálmatas (LORIMIER; KITCHELL, 2002; PASTOR, 2002; SMITH, 2003).

O hemangiossarcoma cutâneo pode ter coloração acinzentada a vermelho escuro, aparência nodular, consistência macia, ausência de cápsula ou ulceração e estar na derme ou infiltrado no tecido adjacente (CHUN, 1999; MACEWEN, 2007).

Os pacientes com hemangiossarcomas dermais ou hipodermais devem ser avaliados por meio de hemograma, perfil bioquímico sérico, urinálise, radiografia torácica, ecocardiograma e ultrassonografia abdominal, pois o prognóstico varia de acordo com a classificação (Tabela 1) (CHUN, 1999; LORIMIER; KITCHELL 2002; PASTOR, 2002).

Tabela 1. Estágio do tumor e prognóstico para cães com hemangiossarcoma dérmico ou hipodérmico.

\begin{tabular}{cll}
\hline ESTÁGIO & \multicolumn{1}{c}{ DESCRIÇÃO } & \multicolumn{1}{c}{ PROGNÓSTICO } \\
\hline I & Tumor primário confinado a derme. & $\begin{array}{l}\text { Sobrevida média de 780 dias com } \\
\text { cirurgia apenas }\end{array}$ \\
II & $\begin{array}{l}\text { Tumor primário envolvendo hipoderme com ou sem } \\
\text { envolvimento da derme; sem envolvimento muscular. }\end{array}$ & $\begin{array}{l}\text { Sobrevida média de 172 dias com } \\
\text { cirurgia apenas. } \\
\text { Sobrevida média de 307 dias com } \\
\text { cirurgia apenas. }\end{array}$ \\
\hline
\end{tabular}

(CHUN, 1999). Londrina, 2008.

Hemangiossarcoma cutâneo invasivo pode resultar em metástase através da via hematógena ou implantação transabdominal, principalmente no fígado, omento, mesentério e pulmões (LORIMIER; KITCHELL, 2002; SMITH, 2003; MACEWEN, 2007). A maioria dos animais (80\%) apresenta metástase no momento do diagnóstico (PASTOR, 2002; MACEWEN, 2007).

O tratamento baseia-se em cirurgia agressiva, associada à quimioterapia (MACEWEN, 2007), principalmente com doxorrubicina, além de radioterapia e imunoterapia (CHUN, 1999; LORIMIER; KITCHELL, 2002; PASTOR, 2002; SMITH, 2003). O prognóstico é bom quando o hemangiossarcoma estiver confinado à derme e for possível a excisão cirúrgica completa, sendo reservado a ruim nas demais localizações (LORIMIER; KITCHELL, 2002; SIMON, 2006).

O objetivo do presente trabalho é descrever a invasão do canal medular lombar e consequente desenvolvimento de paraplegia aguda em um cão com hemangiossarcoma cutâneo paravertebral.

\section{Relato do Caso}

Um cão macho, scotish terrier, de sete anos foi atendido no Hospital Veterinário da Universidade Estadual de Londrina por apresentar claudicação de membro posterior direito há sete dias que evoluiu para impotência de membros posteriores 
em 24 horas, mesmo após administração de antiinflamatórios por uma semana. O proprietário relatou ainda a presença de um nódulo em região dorso lombar direita de crescimento lento, com dois meses de evolução. Constatou-se que este apresentava 7,0 X 3,0 cm de diâmetro, era firme e aderido à musculatura na região da quarta vértebra lombar.

Ao exame neurológico verificou-se síndrome toracolombar grau $\mathrm{V}$ (paraplegia, perda da sensibilidade profunda e perda do controle da micção e defecação), existindo grande hiperestesia vertebral cranialmente ao nódulo. $\mathrm{Na}$ radiografia toracolombar lateral observou-se uma imagem sugestiva de subluxação entre a quarta e quinta vértebras lombares com discreta lise óssea (Figura 1A). Foi realizada mielografia, visualizando-se interrupção na coluna de contraste entre as vértebras torácicas 11 e 12 (Figura 1B).

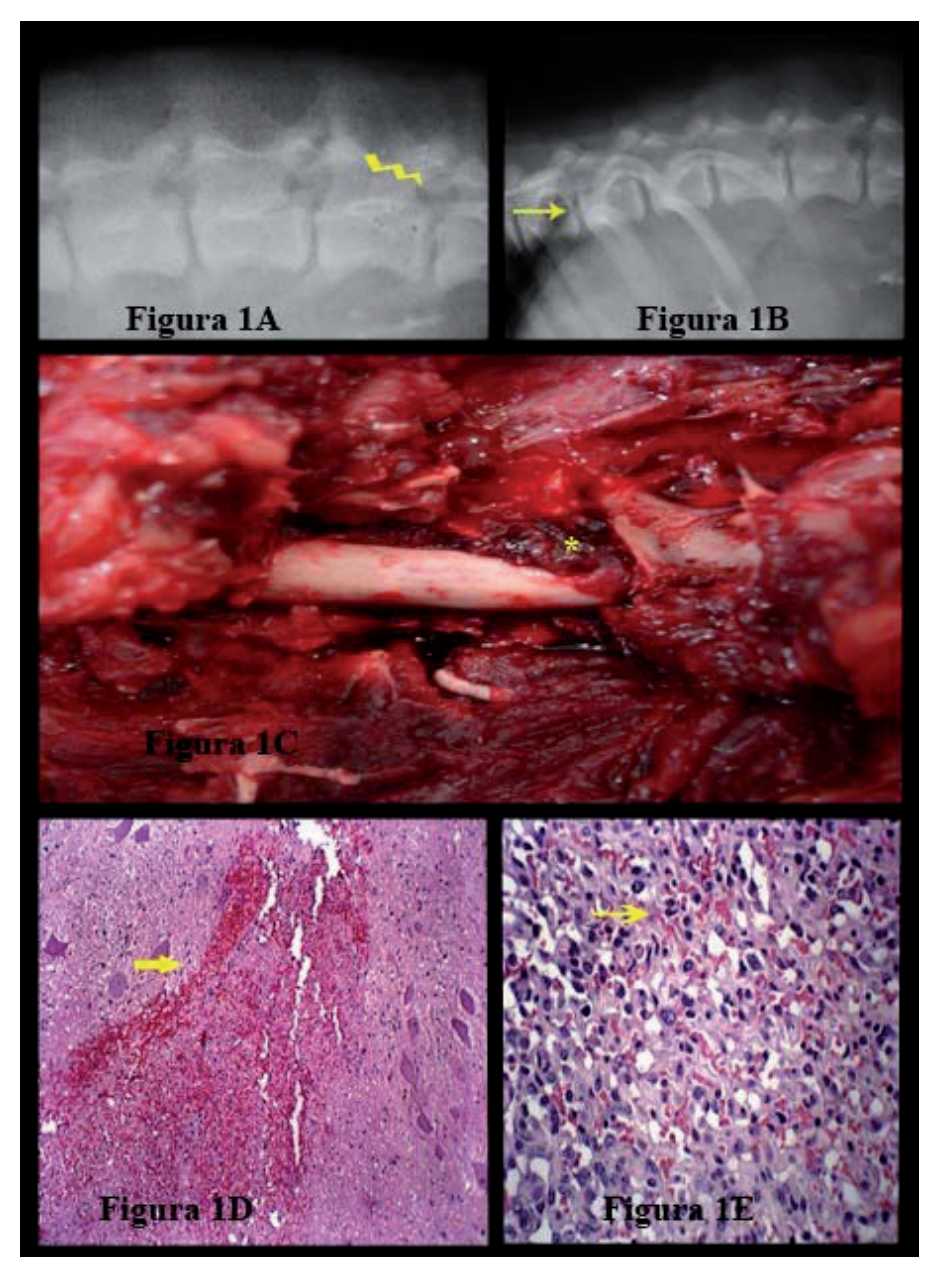

Legenda:

Figura 1A: Radiografia toracolombar em projeção lateral. Observa-se imagem sugestiva de subluxação entre a quarta e quinta vértebras lombares com discreta lise óssea (raio).

Figura 1B: Mielografia. Pode-se observar interrupção na coluna de contraste entre as vértebras torácicas 12 e 13 (seta).

Figura 1C: Imagem realizada durante o período trans-operatório, após a realização de laminectomia na região da quarta vértebra lombar. Observa-se desvio da medula espinhal para o lado esquerdo, devido à presença de uma massa de coloração avermelhada originada do lado direito (asterisco).

Figura 1D: Fotomicrografia da medula na região subjacente à infiltração tumoral. Observa-se hemorragia focalmente extensa na região dorsal da substância cinzenta (seta). HE (10x).

Figura 1E: Fotomicrografia do hemangiossarcoma. Observam-se células de elevada anaplasia, fusiformes e dispostas em lumens, com pequena quantidade de hemácias no seu interior (seta). HE (40x). 
Devido ao resultado do exame contrastado, procedeu-se à hemilaminectomia entre as vértebras T11 e T12, onde não foi constatada compressão medular e assim, procedeu-se a ampliação caudal da abertura da lâmina vertebral. Na região da quarta vértebra lombar observou-se desvio da medula espinhal para o lado esquerdo, devido à presença de uma massa de coloração avermelhada originada do lado direito, diagnosticando-se infiltração tumoral em vértebras lombares com compressão medular (Figura 1C). Como não era possível a retirada do tumor com ou sem margem adequada, independente de sua origem, foi decidido em conjunto com os proprietários a realização de eutanásia no período transoperatório.

No exame histopatológico da massa, verificouse a presença de células de elevada anaplasia, fusiformes e dispostas em lumens, com pequena quantidade de hemácias no seu interior e figuras de mitose freqüentes (Figura 1D), classificando-se o tumor como hemangiossarcoma. Havia hemorragia focalmente extensa na região dorsal da substância cinzenta e degeneração walleriana acentuada nos funículos ventrais da medula (Figura 1E).

\section{Discussão}

Em cães com paraplegia os diagnósticos diferenciais devem ser considerados de acordo com a apresentação clínica em agudo não progressivo, agudo progressivo e crônico progressivo ou não (BRAUND, 1994). O presente relato pode ser classificado como agudo progressivo, pois havia histórico do aparecimento de claudicação do membro posterior direito uma semana antes do atendimento e logo após houve o desenvolvimento de paraplegia súbita. Assim, a suspeita inicial de acordo com a apresentação, raça e idade foi de doença do disco intervertebral (DDIV). Knowles et al. (1977) descreveram um caso de hemangiossarcoma pulmonar com infiltração em coluna torácica com evolução para paraparesia em quatro horas, cujo diagnóstico diferencial inicial também foi DDIV.
Segundo os autores Chun (1999), Lorimier; Kitchell (2002) e Macewen (2007), os sinais agudos observados em animais com hemangiossarcoma são consequência de ruptura de vasos com consequente hemorragia, porém neste caso as alterações neurológicas agudas podem ter sido sinais agudos resultantes da infiltração tumoral na medula espinhal. Quando o tumor infiltra-se na musculatura, o paciente poderá apresentar claudicação aguda, aumento de volume muscular firme e edema na porção distal (MACEWEN, 2007).

Devido à suspeita de DDIV e ausência de dor profunda, o indicado era a intervenção cirúrgica imediata para amenizar os danos à medula espinhal (OLBY et al., 2003). Na mielografia o bloqueio do contraste ocorreu seis espaços craniais à localização da massa e da imagem lítica observada, contribuindo para a suspeita errônea de DDIV. Assim, procedeu-se a hemilaminectomia nas vértebras torácicas, e posteriormente na região lombar, na qual se observou a infiltração da massa tumoral no canal vertebral lombar, justamente entre a quarta e quinta vértebras lombares, local em que se observou imagem sugestiva de subluxação com discreta lise óssea na radiografia toracolombar lateral. Esse fato está de acordo com uma descrição de hemangiossarcoma ósseo, no qual por meio do exame radiográfico verificou-se tendência mais lítica do que proliferativa (MACEWEN, 2007).

Ao sofrer uma compressão ou trauma, a microcirculação da superfície medular é alterada, resultando em paralisia vasomotora com estase intravascular em seus capilares e vênulas, ocorrendo resposta inflamatória na parede desses vasos, o que resulta em edema medular (TATOR, 1972). $\mathrm{Na}$ mielografia, esse edema impede a progressão e o preenchimento do espaço subaracnóide pelo contraste (GOPAL; JEFFERY, 2001), o que pode no presente relato justificar a interrupção tão cranial do contraste na coluna em relação ao local da infiltração tumoral. 
A tomografia computadorizada ou a ressonância magnética, quando disponíveis, ajudam a determinar a extensão da invasão local (LORIMIER; KITCHELL, 2002), porém nesse caso, não foi possível realizá-las e assim a invasão tumoral na coluna vertebral só foi confirmada durante a cirurgia. MORGAN et al. (2007) relataram um lipoma infiltrativo em coluna torácica em um cão com paraparesia aguda cujo diagnóstico foi feito através da ressonância magnética e enfatizam sua importância no planejamento cirúrgico. Contudo, Tucker etal. (2000) relataram um hemangiossarcoma primário em iliopsoas de cão, no qual concluíram que a ultrassonografia poderia ser apropriada para avaliação de massas musculares, sendo um exame mais acessível e econômico que a ressonância magnética.

Pode-se suspeitar de hemangiossarcoma pela história e apresentação clínica, porém o diagnóstico definitivo só é possível com o exame histopatológico (BROWN; PATNAIL; MACEWEN, 1985; PASTOR, 2006; MACEWEN, 2007).Assim,nestecaso, pelaanálisehistopatológica da massa, diagnosticou-se hemangiossarcoma que foi classificado em estágio III conforme descrição de CHUN (1999) (Tabela 1).

Simon (2006) relata que em um cão com hemangiossarcoma cutâneo infiltrativo em abdome lateral direito, não foi possível a remoção cirúrgica com margem e apesar da realização de radioterapia e quimioterapia, conseguiu-se uma sobrevida de um ano, ocorrendo recidiva do tumor. Brown, Patnail e Macewen (1985) concluíram em uma análise retrospectiva de 104 casos, que a sobrevida dos cães com hemangiossarcoma primário foi menor que quatro meses, mesmo com ressecção cirúrgica.

O hemangiossarcoma cutâneo é mais descrito em cães pouco pigmentados ou de pelos claros podendo estar associado à exposição aos raios ultravioletas (LORIMIER; KITCHELL, 2002; SMITH, 2003; MACEWEN, 2007). O caso descrito apresentava pelo escuro, diferindo da literatura. Os locais mais frequentemente acometidos são aqueles com menor revestimento piloso, como o abdome ventral ou pele prepucial (CHUN, 1999; LORIMIER; KITCHELL, 2002; MACEWEN, 2007), contudo, no presente relato o tumor estava localizado na região lombar paravertebral.

\section{Conclusão}

Conclui-se que o hemangiosarcoma cutâneo, a exemplo de outras neoplasias, pode ter comportamento infiltrativo em direção ao canal medular e deve ser considerado no rol de diagnósticos diferenciais em cães apresentando paraplegia aguda. No caso aqui relatado, devido à apresentação aguda, não foi possível realizar citologia ou histologia para diagnóstico previamente à realização da cirurgia.

\section{Referências}

BRAUND, K. G. Clinical Syndromes in Veterinary Neurology. 2. ed. St. Luis: Mosby, 1994, 477 p.

BROWN, N. O.; PATNAIK, A. K.; MACEWEN, E. G. Canine hemangiosarcoma: Retrospective analysis of 104 cases. Journal of the American Veterinary Medical Association, v. 186, n. 1, p. 56-58, Jan. 1985.

CHUN, R. Feline and Canine Hemangiosarcoma. Compendium on Continuating Education for Practioners Veterinarians, v. 21, n. 7, p. 622-629, July 1999.

GOPAL, M. S.; JEFFERY, N. D. Magnetic resonance imaging in the diagnosis and treatment of a canine spinal cord injury. Journal of Small Animal Practice, v. 42, n.1, p. 29-31, Jan. 2001.

KNOWLES, J. O.; BLOCK, J. H.; SHAFFER, P. A.; GRISWOLD, M. L. Posterior paresis caused by extension of hemangiosarcoma from lungs to thoracic vertebrae of a dog. Journal of the American Veterinary Medical Association, v. 171, n. 15, p. 544-545, Sept. 1977.

LORIMIER, L. P.; KITCHELL, B. E. How to manage patients with hemangiosarcoma. Veterinary Medicine, $\mathrm{v}$. 97, n. 1, p. 46-57, Jan. 2002.

MACEWEN, E.G. Hemangiosarcoma. In: WITHROW, S. J.; MACEWEN, E. G. Small Animal Clinical Oncology. 4.ed. Philadelphia : W. B. Saunders Company. p.785794, 2007. 
MORGAN, L. W.; TOAL, R.; SIEMERING, G.; GAVIN, $\mathrm{P}$. Infiltrative lipoma causing spinal cord compression in a dog. Veterinary Radiology \& Ultrasound, v. 48, n. 1, p. 35-37, 2007.

OLBY, N.; LEVINE, J.; HARRIS, T.; MUÑANA, K.; SKEEN, T.; SHARP, N. Long-term functional outcome of dogs with severe injuries of the thoracolumbar spinal cord: 87 cases (1996-2001). Journal of the American Veterinary Medical Association, v. 222, n. 1, p. 15021503, June. 2003.

PASTOR, J. Canine hemangiosarcoma. Clinical update. 27 WSAVA- World Animal Veterinary Association Congress, Granada, 2002.
SIMON, D. Multimodality treatment of subcutaneous hemangiosa. 2006. Disponível em: <http://www.ivis. org>. Acesso em: 11 Jan. 2006.

SMITH, A. N. Hemangiosarcoma in dogs and cats. The Veterinary Clinics Small Animal Practice, v. 33, n. 3, p. 533-552, 2003.

TATOR, C. H. Acute spinal cord injury: a review of recent studies of treatment and pathophysiology. Canadian Medical association Journal, v.107, n. 2, p. 143-150, July 1972.

TUCKER, D. W.; OLSEN, D.; KRAFT, S. L.;ANDREWS, G. A.; GRAY, A. P. Primary hemangiosarcoma of the iliopsoas muscle eliciting a peripheral neuropathy. Journal of the American Animal Hospital Association, v. 36, p. 163-167, mar./apr. 2000. 